\title{
Monocular 3D Head Reconstruction via Prediction and Integration of Normal Vector Field
}

\author{
Oussema Bouafif $^{1,2}$, Bogdan Khomutenko ${ }^{1}$ and Mohamed Daoudi ${ }^{2}$ \\ ${ }^{1}$ MCQ-Scan, Lille, France \\ ${ }^{2}$ IMT Lille Douai, Univ. Lille, CNRS UMR 9189 CRIStAL, Lille, France \\ oussema.bouafif@univ-lille.fr,b.khomutenko@mcq-scan.com,mohamed.daoudi@imt-lille-douai.fr
}

\begin{abstract}
Keywords: 3D Head Reconstruction, Face Reconstruction, Monocular Reconstruction, Facial Surface Normals, Deep Learning, Synthetic Data.

Abstract: $\quad$ Reconstructing the geometric structure of a face from a single input image is a challenging active research area in computer vision. In this paper, we present a novel method for reconstructing 3D heads from an input image using a hybrid approach based on learning and geometric techniques. We introduce a deep neural network trained on synthetic data only, which predicts the map of normal vectors of the face surface from a single photo. Afterward, using the network output we recover the 3D facial geometry by means of weighted least squares. Through qualitative and quantitative evaluation tests, we show the accuracy and robustness of our proposed method. Our method does not require accurate alignment due to the image-to-image translation network and also successfully recovers 3D geometry for real images, despite the fact that the model was trained only on synthetic data.
\end{abstract}

\section{INTRODUCTION}

In the last decades, 3D face models have been employed in several applications of Computer Vision. Unlike 2D face images, the three-dimensional face model reconstruction can encounter different problems, such as variations in poses and illumination (Abate et al., 2007). A 3D face model has the potential to achieve state-of-the-art performances on applications such as gender classification (Han et al., 2009), facial animation (Thies et al., 2016) and face recognition (Blanz and Vetter, 2003).

Originally, the problem has been treated using the following techniques. A large part of the proposed solutions use facial landmarks, that is, a set of automatically detected key points on the face, which can be used as a guideline for the reconstruction process. Many methods are based on optimization algorithms and use the 3D Morphable Model (3DMM) proposed by Blanz and Vetter (Blanz and Vetter, 1999), which is a statistical model of texture and shape. Some approaches are based on structure from motion, optical flow or shape from shading procedures (Kemelmacher-Shlizerman and Basri, 2010). Despite the fact that the use of these elements allows us to perform the reconstruction, there are some difficult cases for such methods. They are sensitive to light condi- tions, reflections, shadows, and image quality.

Another way to separate 3D face reconstruction methods is to take into account the number of input images. Monocular methods have a significant drawback which is the fact of being unable to recover precise geometric measurements with a single frontal view. In addition, the local details that characterize the shape of the face between all surfaces are complex to grasp, resulting in very similar reconstructions from one subject to another. However, with multiview-based methods, it is possible to have a more faithful 3D reconstruction since we exploit the geometric constraints of several images in different views. However, these methods frequently produce noisy results.

More recently, solutions to address this problem have changed with Convolutional Neural Networks $(C N N s)$ and request only a single image as input (Richardson et al., 2017; Dou et al., 2017). But one of the most known difficulties to apply neural networks is the lack of 3D faces data sets. To answer this need, many approaches propose to use synthetic data or 3D models fitted by using one of the methods cited above. In some cases, when the training set is limited, endto-end learning systems tend to perform worse than geometric methods.

In this paper, we propose a hybrid method com- 


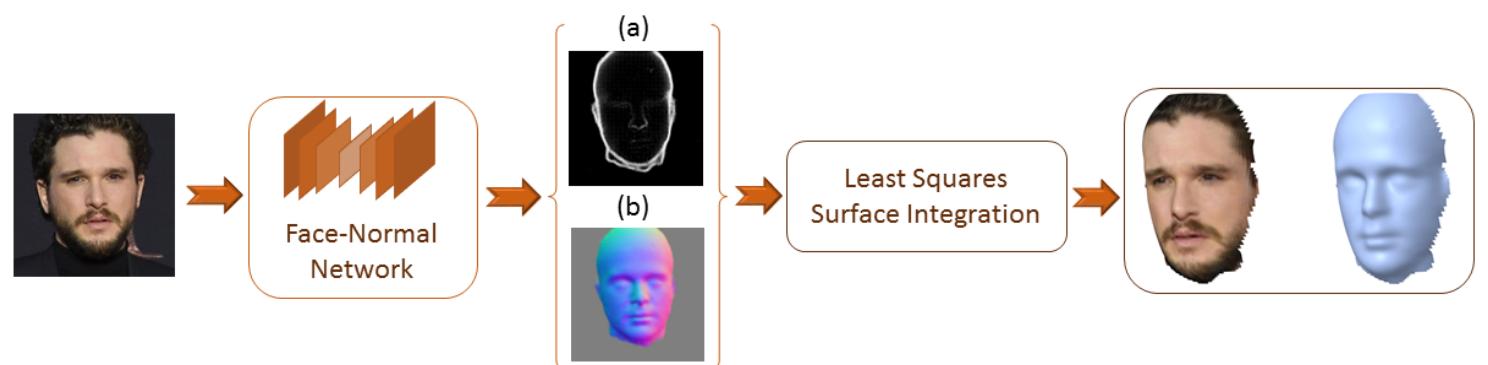

Figure 1: The pipeline of our proposed method. Given an input facial image, we estimate two different maps (magnitude of depth gradient map $W(\mathrm{a})$, normal surface map $N(\mathrm{~b}))$ through a network which was trained using a fully synthetic data set. Using these generated maps, we reconstruct the 3D facial shape by a weighted least squares normal integration technique where $W$ acts as a weight map.

posed of a learning-based approach and a geometric one that is capable of reconstructing face surface from an input facial image. In the first stage, from the learning approach, we show that a Generative Adversarial Model (GAN) can translate a facial image into two maps: normals of facial surface $(N)$ and gradient magnitude $(W)$. Using these maps in a weighted least squares $(W L S)$ technique, we retrieve the 3D facial surface.

The main contributions of this paper are:

- We use a fully synthetic data set of 3D human heads composed of different elements including 3D faces geometry from the LYHM (Dai et al., 2017 ) model, hair models from (Hu et al., 2015) database and different faces textures, eye colors and eyeglass patterns (Section 3.1),

- A Generative Adversarial Model $(G A N)$ from $(\mathrm{Su}$ et al., 2018) adapted to predict different images from the input face image that will be used during the reconstruction step (Section 3.2),

- A more reliable head reconstruction using a novel normal integration technique based on a weighted least squares method (Section 3.3.

\section{RELATED WORK}

In this section, we review works on $3 \mathrm{D}$ face reconstruction methods, prediction and integration of normals.

The different components of the human face may be divided into two broad groups, one for the lowdetail geometry (e.g., nose, cheek, forehead) and the other one for the high-detail geometry (e.g., wrinkles, eyebrows, beards, and pores). Methods such as multiview geometry (Furukawa and Ponce, 2009) and structure-from-motion (Gonzalez-Mora et al., 2010), which are based on reconstruction from multiple images, can recover the low-detail geometry features.
However, to be able to capture the high-detail geometric features, successful solutions rely on professional capture systems such as 3D laser scans or highprecision multi-view light stage systems such as those used in (Ghosh et al., 2011). In reality, these kinds of methods require a significant investment in space, time and finances for the large setup, powerful light sources, as well as extensive calibrations of the position and direction of the light sources.

Monocular 3D face reconstruction methods: Generally, the best-known methods that use few images, or a single image, as the input to reconstruct a 3D face are based on the work of Blanz and Vetter (Blanz and Vetter, 1999), who proposed the 3D Morphable Model (3DMM). The model consists of a separate shape model and an albedo model, constructed using Principal Component Analysis (PCA). The key idea behind the 3DMM is that, given a sufficiently large data set of 3D faces, one can accurately reconstruct any new shape and texture as a linear combination of the shapes and textures of the 3D faces in the data set. The use of 3DMM allows us to reconstruct a new 3D face from one or more images by finding the linear combination of the statistical model bases that best fits the given 2D image(s). For example, (Amberg et al., 2008) fit an expression-invariant 3DMM to noisy laser scans using an Iterative ClosestPoint (ICP) registration method. In (Zollhöfer et al., 2011), authors propose to fit a 3DMM directly to the aggregated data from a consumer depth camera. Their idea was to deform the mean shape of a 3DMM to the aggregated depth data using the non-rigid registration method from (Sumner et al., 2007). Most other methods are based on landmarks (Booth et al., 2017), edges (Bas et al., 2016) and local image features ( $\mathrm{Hu}-$ ber et al., 2015). Recently, Convolutional Neural Networks (CNNs) were used with 3DMMs to reconstruct 3D faces from a single input photo. (Tran et al., 2017) fit the 3DMM to the images in a data set and com- 
bined the shape and texture vectors that corresponded to images of the same person. They proposed using regression methods to obtain the 3DMM shape and texture parameters directly from an input photo. (Dou et al., 2017) proposed UH-E2FAR, an end-toend 3D face reconstruction method based on deep neural networks. They introduced two key components - a fusion-CNN and a multi-task learning loss. With both components, they divided 3D face reconstruction into two sub tasks - predicting the neutral 3D facial shape and the expression parameters of a 3DMM - using a single frontal image from each person. (Richardson et al., 2017) proposed an end-toend approach composed of two connected networks (CoarseNet and FineNet) to produce coarse and fine details of facial shape. (Sela et al., 2017) presented an algorithm which employs an Image-to-Image translation network that jointly maps the input image to a depth image and a facial correspondence map. A model-free approach was proposed by (Feng et al., 2018a) which learns 3D face curves from horizontal and vertical epipolar plane images of a light filed images using a densely connected network (FaceLFnet). Produced curves are combined together to obtain a more accurate combined 3D point cloud. In (Feng et al., 2018b), an encoder-decoder structure was used to learn a transfer function between an input RGB image and the UV position map, which was a 2D representation designed to record the 3D shape of a complete face in UV space.

Prediction of normals: Normal maps are used in various graphics applications like 3D shape reconstruction or adding details to allow rendering of surfaces to be more realistic. But producing highquality normal maps for complex objects represents a challenging task. To resolve this problem, several learning-based works have been proposed. Part of these applications were devoted to the generation of normal maps based on sketches with deep neural networks. In (Su et al., 2018) work, an interactive method for normal map generation from sketch input was proposed where the U-Net (Ronneberger et al., 2015) architecture was adopted in a conditional GAN framework. (Lun et al., 2017) used ConvNet network to predict depth and normal maps from multi-view sketches, and then combine outputs into a 3D point cloud via energy minimization. Another sketch-based work was proposed by (Hudon et al., 2018), were they present a way of predicting high-resolution normal maps directly without any user annotation or interaction. Using a multi-scale representation of their input images, they ensure the efficiency and quality of produced data. Another category of methods was proposed to predict the normal map from different objects or (outdoor/indoor) scenes. Several approaches have been addressed by (Bansal et al., 2016) with a skip-network model, (Qiu et al., 2019) with a jointly predicted depth and surface normal from a single image, (Wang et al., 2015) with a network to estimate both local and global normal map estimation. Similarly to our work, (Trigeorgis et al., 2017) proposed a 3D face reconstruction method based on integration normal method where facial normal map is produced by a fully-convolutional network. Our proposed pipeline is different from (Trigeorgis et al., 2017) in three important aspects: firstly, we use a fully synthetic data set of 3D heads, whereas the (Trigeorgis et al., 2017) data set was composed of various data sets mainly limited to the facial part of the head. Secondly, (Trigeorgis et al., 2017) explored various DCNN architectures whereas we use the symmetric skipping network (U-Net) (Ronneberger et al., 2015) with a discriminator (Su et al., 2018), a common feature of Generative Adversarial Networks. Thirdly, (Trigeorgis et al., 2017) used the standard FrankotChellappa method (Frankot and Chellappa, 1988a) to recover $3 \mathrm{D}$ facial shape from predicted normals. We use a weighted least square method along with magnitude depth gradient maps as a way to improve the reconstruction quality in the neighborhood of depth discontinuities. Furthermore, we provide quantitative evaluation of the reconstruction precision, performed on the BU-3DFE (Yin et al., 2006) data set.

Integration of normal: Various approaches have suggested to estimate the depth maps from normals for a long time and they are generally classified in several groups. In (Frankot and Chellappa, 1988b) and (Simchony et al., 1990), Discrete Fourier Transform and Discrete Cosine transform-based methods are proposed. Some other basis variants have been proposed where they use shapelets (Kovesi, 2005), wavelet (Hsieh et al., 1995) or Dirac delta functions (Karaçalı and Snyder, 2003). The reconstruction via the Poisson equation (Simchony et al., 1990) is probably the most well-known technique. This approach uses the $\ell^{2}$ norm since it is assumed that the residual gradient follows a normal distribution. And so, the problem is quadratic and therefore admits a unique and simple solution. On the other hand, the $\ell^{2}$ norm does not support the presence of outliers, which can produce deformed surfaces. In this context, Agrawal et al., 2006) propose a general framework to extend the Poisson equation. Other approaches known as regularization methods (Terzopoulos, 1988; Harker and Oleary, 2015) have been proposed and attempt to smooth depth gradients under certain criteria. Fi- 
nally, the latest approaches are weighted-based, and they use the constraint partially by a weighting map where weight values were planned to deal with local discontinuity. Similarly to these works Quéau and Durou, 2015; Wang et al., 2012), we propose the use of a weighting map generated from a deep neural network model. Recently, (Xie et al., 2019) proposed an approach based on a discrete framework for discontinuity preservation where two normal incompatibility features and an efficient discontinuity detection scheme were introduced. More normals integration state-of-art methods were explained in (Quéau et al., 2018).

\section{PROPOSED METHOD}

In this section, we describe the details of our proposed framework as illustrated in Fig. 1. Our method takes a facial image as input and the network produces two outputs which are aligned with the input image: an estimated normal surface $N$ and an image of estimated magnitude of the depth gradient $W$. All of these outputs are used in a $3 \mathrm{D}$ reconstruction algorithm guided by $W$ to recover the 3D surface of the face. Firstly, we introduce the synthetic data set generation method used to produce data for the training stage in Section 3.1. In Section 3.2 we describe our network model architecture which was used in (Su et al., 2018) and illustrated in Fig. 3 Finally, the details of our reconstruction stage are explained in Section 3.3 .

\subsection{Synthetic Data Generation}

Compared to (Trigeorgis et al., 2017) work, which was based on a mix of synthetic and real data to train the network, our proposed model has been trained only on synthetic data. To do that, we have set up a synthetic data generator composed of several parts that gives us a complete human head model. We used for the 3D human head model a 3DMM craniofacial model proposed by (Dai et al., 2017). The Liverpool York Head Model (LYHM) (Dai et al., 2017) has been used to provide a parametric model to synthesize heads with a known ground truth geometry. Its composed of two parametric models: the shape and the texture. Changing the shape and the texture parameters can create different subjects.

In our work, we use only the shape part which was described with a linear model that is used to generate novel 3D head examples as follows :

$$
X=X_{0}+W y
$$

Where $X$ is the 3D head, $X_{0}$ the mean face shape, $W$ is the principal components of the shape model,
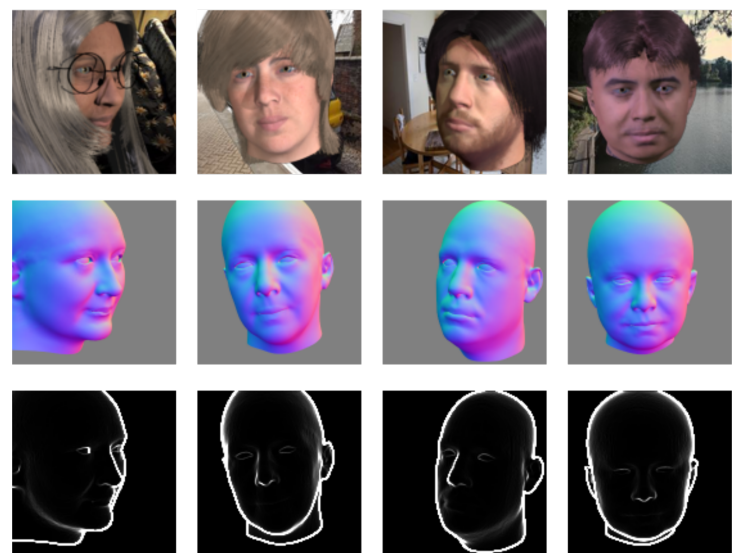

Figure 2: Training data samples. From top to bottom: Synthetic facial images. Normal surface maps $N$. Gradient Magnitude maps $W$.

and $y$ is the corresponding coefficient vector shape. Using this model, an infinite number of synthetic faces can be generated by choosing a pair of parameters from a normal distribution $y \sim N(0,1)$. For the hair we use different models from the (Hu et al., 2015) data set with different colors and the Kajiyakay model (Kajiya and Kay, 1989) for the hair lighting rendering. We also use different male and female face textures directly mapped with the 3D head model and we align the head with a 3D eyes model where we randomly change the color of the iris for each head model. Finally, we also simulate six different 3D glasses models to create some occlusions. Once the different 3D components were aligned, random heads are generated under various illumination conditions, shadows, poses, scales, coefficients for physicallybased materials. Finally, to make the model insensitive to the background, a random background image taken from the COCO data set (Lin et al., 2014) is added. Some examples of the training data set used for this work are shown in Fig. 2

Once the final 3D head model has been generated as detailed above, we compute the normal surface only for the head and eyes models by using the angle weighted method from (Klasing et al., 2009) as written in (2). Normal values $n_{i}$ are calculated for each vertex location $p_{i} \in \mathrm{Re}^{3}$, given the set of vertices $\left\{q_{i, 1}, q_{i, 2}, \ldots, q_{i, k}\right\}$ that are adjacent to $p_{i}$.

$$
\begin{gathered}
n_{i}=\frac{1}{k} \sum_{j=1}^{k} \omega_{j} \frac{\left[q_{i, j}-p_{i}\right] \times\left[q_{i, j+1}-p_{i}\right]}{\left|\left[q_{i, j}-p_{i}\right] \times\left[q_{i, j+1}-p_{i}\right]\right|}, \\
\omega_{j}=\arccos \left(\frac{\left\langle q_{i, j}-p_{i}, q_{i, j+1}-p_{i}\right\rangle}{\left|q_{i, j}-p_{i}\right|\left|q_{i, j+1}-p_{i}\right|}\right) .
\end{gathered}
$$

The choice fell on the use of normals for various reasons. First, components of normal field define lo- 
cal geometric properties and hence disentangled from one another across certain distances in the sense that we can predict them completely independently, in contrast to the depth values which should be predicted all together. That is, without knowing the depth value of the tip of the nose, for example, one cannot predict the value for the eyes. Second, normals are invariant to translation and scaling. To improve the quality of $3 \mathrm{D}$ reconstruction, the generation of gradient magnitude map $|\nabla f(x, y)|$ from the pixel-wise depth image is proposed (third row in Fig. 2). The effect of using this information is analysed in Section 3.3

\subsection{Network Structure}

The proposed GAN architecture is based on (Su et al., 2018) work, where a network has been trained to map the normal surface map from a sketch and a binary point mask inputs. Some changes have been made to this network to adapt it to our problematic (more details in Fig. 3).

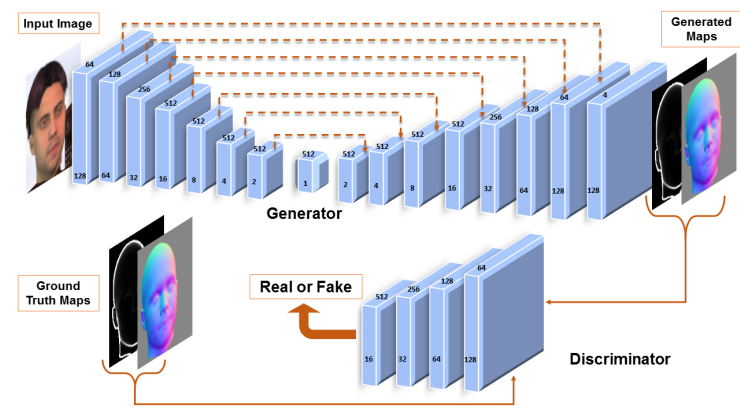

Figure 3: Our neural network architecture aims to generate two maps given facial input image. The input training data as is shown on the left is composed of a facial input image and two ground-truth maps: $N$ and $W$. The encoderdecoder's input is the image of the face, while at the output it produces two different maps (shown on the right). After that, we inject ground truth and generated maps together with the facial image as the input of discriminator. In this stage, we check if the generated maps are real or fake, so we encourage the encoder-decoder to produce more realistic maps according to face image input. The spatial size and the number of layers are indicated in and above each block, respectively.

The model is composed of a encoder-decoder network and a discriminator, proposed in Ronneberger et al., 2015). All training images have $128 \times 128$ pixels size. As an input, we stack three images, the facial image (RGB), $N$ (three channels) and $W$ (single channel), while we have only two generated maps.

The encoder has the same elements as the discriminator (discussed below), the decoder is composed of ReLU activation function, deconvolution, batch normalization, and a dropout unit. To reduce the informa- tion loss between successive layers, we use a symmetric connection between the encoder and the decoder (Ronneberger et al., 2015). Therefore, we concatenate each layer of the encoder with the corresponding channel in the decoder. The encoder-decoder is composed of 16 layers. In the output layer, tanh is used as an activation function, since $N$ lies in the range $[-1,1]$. The discriminator is composed of 4 layers, and it is inspired by the encoder. Each layer is composed of: convolution, batch normalization, and ReLU activation function. We adopt the loss function used in $(\mathrm{Su}$ et al., 2018) for our purposes. Our objective function becomes:

$$
\begin{gathered}
F=\mathbb{E}_{x \sim p_{\text {data }}, y \sim p_{m}}[D(y / x)]-\mathbb{E}_{\tilde{y} \sim p_{\text {gen }}}[D(\tilde{y} / x)]- \\
\lambda_{1} L_{2}, \\
L_{2}=\mathbb{E}_{y \sim p_{m}, \tilde{y} \sim p_{\text {gen }}}\left[\|y-\tilde{y}\|_{2}\right]
\end{gathered}
$$

Where $x$ represents the input face image, $y$ is the corresponding concatenated input maps, $\tilde{y}$ is the generated outputs. $P_{\text {data }}, P_{m}$, and $P_{\text {gen }}$ are the distributions of real input data, input map and generated outputs data, respectively. The encoder-decoder loss is mixed with a pixel wised loss $L_{2}$ penalized by $\lambda_{1}$ to measure the difference between the generated maps and the real input maps, and so to supervise the training process.

\subsection{D Face Reconstruction}

Our 3D reconstruction solution is based on the integration of normals guided by the magnitude of the depth gradient, to improve the reconstruction precision in the presence of discontinuities. For this, we retrieve the two output maps $W$ and $N$ from the generative model where:

- $W: \mathbb{R}^{2} \rightarrow \mathbb{R}$ is the magnitude of depth gradient,

- $N: \mathbb{R}^{2} \rightarrow \mathbb{R}^{3}$ is the normal surface map.

Thenceforth, we compute depth gradient $G$ based on $N$ as described in the following:

$$
\begin{gathered}
G: \mathbb{R}^{2} \rightarrow \mathbb{R}^{2} \\
G(u, v)=\left[\begin{array}{l}
p(u, v) \\
q(u, v)
\end{array}\right]=\left[\begin{array}{l}
-\frac{N_{x}(u, v)}{N_{z}(u, v)} \\
-\frac{N_{y}(u, v)}{N_{z}(u, v)}
\end{array}\right]
\end{gathered}
$$

where $N_{x}, N_{y}$, and $N_{z}$ are the three components of $N$ and $(u, v)$ represents the pixels of the discrete domain $\Omega \subset \mathbb{R}^{2}$. After that, we feed $G(u, v)$ and $W(u, v)$ into a weighted least squares solver defined in a continuous setting as follows:

$$
\underset{h}{\arg \min } \iint_{u, v \in \Omega} w(u, v)\|\nabla h(u, v)-G(u, v)\|^{2} \mathrm{~d} u \mathrm{~d} v
$$




$$
w(u, v)=\frac{1}{1+\lambda W(u, v)}
$$

$w(u, v)$ is the weight term used to enforce conformity of the reconstructed surface with the gradient term near the face discontinuities. In 6 , $\lambda$ is a critical parameter to tune (see details in section 4.2). In this step, we estimate depth map values of a function $h: \mathbb{R}^{2} \rightarrow \mathbb{R}$ within the reconstruction domain $\Omega$ where $G$ and $W$ are defined. In the discrete setting, our minimization problem is formulated as below:

$$
\begin{array}{r}
\underset{h}{\arg \min } \sum_{u, v \in \Omega} w_{u+0.5, v}\left(h_{u+1, v}-h_{u, v}-p_{u+0.5, v}\right)^{2} \\
+w_{u, v+0.5}\left(h_{u, v+1}-h_{u, v}-q_{u, v+0.5}\right)^{2}
\end{array}
$$

Where $(u+0.5, v)$ and $(u, v+0.5)$ are the average points between two successive pixels along the horizontal and vertical axes respectively. For example, gradient between pixels is defined in this way:

$$
\begin{aligned}
p_{u+0.5, v} & =\frac{1}{2}\left(p_{u, v}+p_{u+1, v}\right) \\
q_{u, v+0.5} & =\frac{1}{2}\left(q_{u, v}+q_{u, v+1}\right)
\end{aligned}
$$

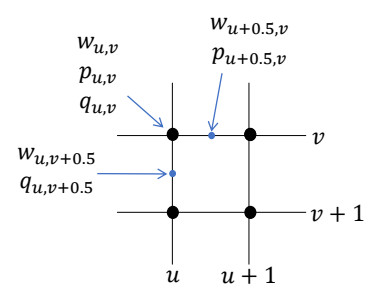

Figure 4: Illustration of our discretization method.

An illustration of our discretization method on a regular square 2D grid is shown in Fig. 4. This is a classical linear least-square problem, we solve it using the Ceres solver (Agarwal et al., ). Evaluation of the reconstruction quality is given in section 4.2

\section{EXPERIMENTAL RESULTS}

This section presents three sets of experiments that were conducted to evaluate the performance of our 3D reconstruction method. Firstly, we discuss the quality of our training model in Section 4.1. Then, we show our contribution to the reconstruction method and the advantage it brings compared to other normals integrations method in Section 4.2 Finally, we verify this by qualitative and quantitative experiments on a known database in Section 4.3
Test Data set: To evaluate the quality of predicted maps from our trained model and also our 3D reconstruction method, we generate a test data set from our generator described in the Section 3.1. This test data set is used in our experiments. It consists of 200 images of people (equitably distributed between males and females) generated with their corresponding ground truth maps that include face images, normal surface maps, gradient magnitude maps, and depth maps. Depth is measured in units equivalent to the pixel size.

\subsection{Training Evaluation}

Table 1: Mask and Normals evaluations for test data set. We show in the top table part our segmentation results using precision and recall percentage. The second part contains angular error results.

\begin{tabular}{|c|c|}
\hline \multicolumn{2}{|c|}{ Mask Evaluation } \\
\hline precision & $93.72 \%$ \\
\hline recall & $98.40 \%$ \\
\hline \hline \multicolumn{2}{|c|}{ Normals Evaluation } \\
\hline Mean & $10.01^{\circ}$ \\
\hline Std & $12.45^{\circ}$ \\
\hline$<10^{\circ}$ & $67.50 \%$ \\
\hline$<20^{\circ}$ & $92.65 \%$ \\
\hline$<30^{\circ}$ & $97.13 \%$ \\
\hline
\end{tabular}

To train the model, we have used 40,000 facial images (20,000 for males and also for females) and their corresponding maps. We train the model for about 1700 epochs with a learning rate of $1 e-4,64$ as a batch size, 500 for $\lambda_{1}$, and using RMSprop optimizer. We also add random blur effect and gaussian noise as data augmentation.

To evaluate our Image-to-Image translation model, we show in Fig. 5 different examples with ground truth and maps produced by our GAN model. We can well notice that with the use of our synthetic data which contain occlusions (hair, glasses, random backgrounds), the network succeeds to separate properly the whole head. In situations where there are covered parts, the network tries predicting a more approximate form of the precise shape hidden by the hair in most cases. Using the test database, we performed some experiments to evaluate the accuracy of our network. Table 1 shows the results indicating a significant percentage of precision and recall, which explains that most of the pixels produced by the network correspond to the pixels of ground truth maps. In a second time, we evaluated the precision on the $N$ maps and for this, we calculated the angular error between the maps of the ground truth and those produced by the network. 


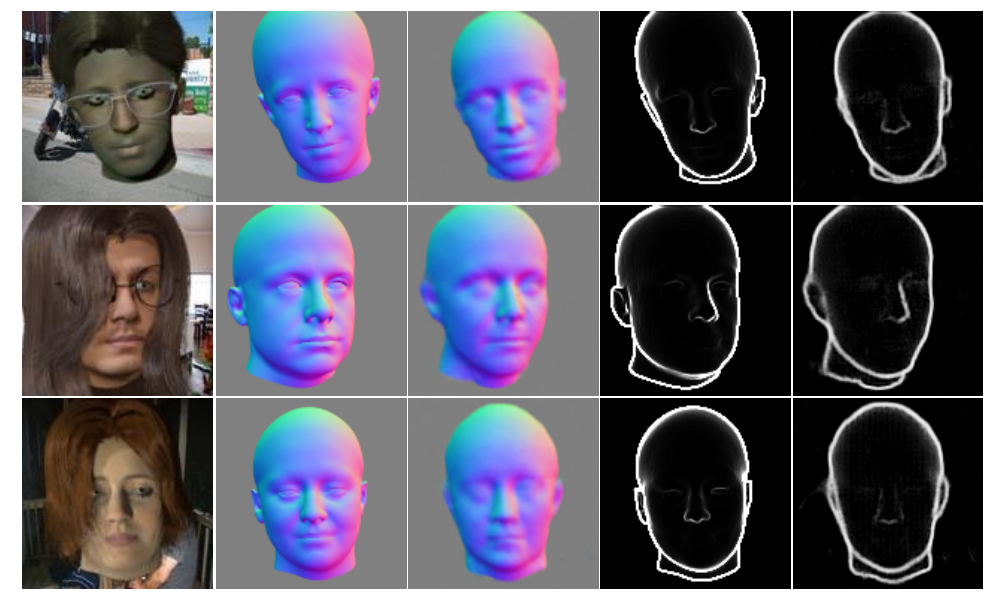

Figure 5: Comparison between ground-truth and estimates $\mathrm{N}$ and $\mathrm{W}$ maps. The first column contains the facial input image, second and fourth columns contain ground-truth maps, third and fifth contain estimated maps.

\subsection{Reconstruction Method Evaluation}

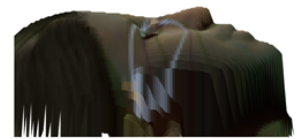

(a)

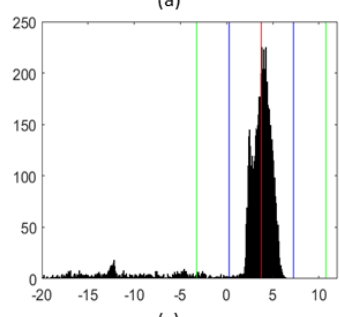

(c)

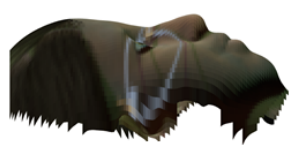

(b)

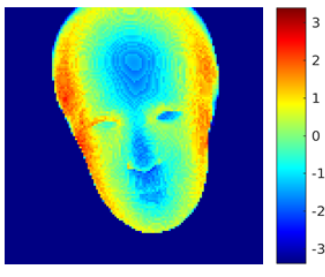

(d)
Figure 6: Example of reconstruction result on synthetic data from test data set

(a) Ground truth surface.

(b) Reconstructed surface.

(c) Histogram of residuals. Red, green and blue lines indicate $M_{v a l}, M_{v a l} \pm \theta$ and $M_{v a l} \pm 3 \sigma_{M}$ respectively.

(d) Heat map with pixels errors after eliminating the bias. The depth error is measured in units equivalent to pixels. The image resolution is $128 \times 128$.

Using the test data set, we evaluate in this step, the efficiency of our proposed method in 3D face reconstruction. To do this, firstly we describe the $\lambda$ parameter tuning procedure to optimize our reconstruction method. Secondly, we show a comparison between the ground truth and the reconstructed depth map using the optimal $\lambda$ value that minimizes the reconstruction error.

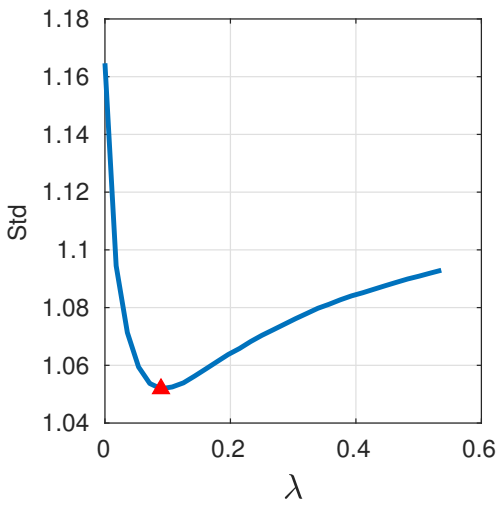

Figure 8: The effect of $\lambda$ on reconstruction accuracy in terms of standard deviation on the test data set.

Once the depth is reconstructed, we compare it to the ground truth, available in the test set. The evaluation procedure used to find the optimal $\lambda$ is implemented as follows:

Step 1: compute the error map between the ground truth and the reconstructed depth maps :

$$
\operatorname{Err}_{M}=d_{G T}-d_{R}
$$

where $z_{G T}$ and $z_{R}$ are the ground truth and reconstructed depths respectively; only the intersection of domains $\Omega_{G T}$ and $\Omega_{R}$ is used.

Step 2: compute median value $M$ of $\operatorname{Err}_{M}$ and given a fixed threshold $\theta=7$, we compute the standard deviation $\sigma_{M}$ of $\operatorname{Err}_{M}$ for values which lie in $\left[M_{v a l}-\theta, M_{v a l}+\theta\right]$.

Step 3: compute the variance $V_{i}$ of $\operatorname{Err}_{M}$ in $\left[M_{v a l}-\right.$ $\left.3 \sigma_{M}, M_{v a l}+3 \sigma_{M}\right]$ range. 


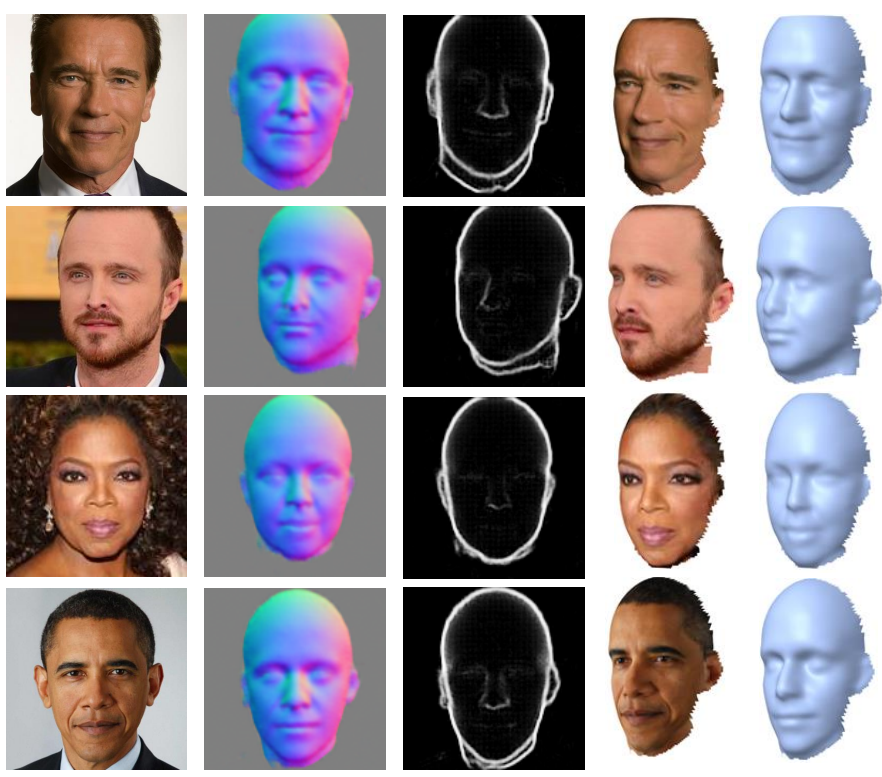

Figure 7: Visual surface reconstruction results from some celebrities facial images. Columns contain in order; input image, estimated $\mathrm{N}$ map, estimated W map and the last two columns contain 3D shape reconstruction.

We perform these steps for all test examples and we compute $\sigma=\sqrt{\sum_{i=1}^{N} \operatorname{Var}_{i} / N}$ for different $\lambda$ values ranging from 0 to 0.3 . The influence of the choice of $\lambda$ on the 3D reconstruction is show in Fig. 8 . The choice $\lambda=0$ corresponds to the least squares solution without weighting. We found that the optimal value is $\lambda=0.1$. This value is used in all experiments hereinafter. In Fig. 6, we illustrate an example from the comparison procedure described above.

\subsection{Final Evaluation}

In order to analyze the performance of the pipeline as a whole, we performed a qualitative experiment on a set of images of celebrities and a quantitative experiment on a 3D facial data set to test its accuracy.

For qualitative analysis, we show in Fig. 7) our results on the image of certain celebrities. One can see that our method produces high-quality results that better fit the overall structure. As we use a full 3DMM head model that also includes the cranial part, our method allows us to recover the 3D model of the head for any visible pixel on the image and it also predicts any area hidden by the hair (third and fourth row in Fig. 7). We also indicate the inferior quality of reconstruction for surfaces containing the neck due to the discontinuity between the face and neck parts (first and second rows in Fig. 7).

Quantitative results are reported in Table 2 For evaluation, we use the BU-3DFE (Yin et al., 2006) data set which contains 3D faces of 100 subjects with seven different expressions and each 3D model has corresponding 2D images. Using only neutral expressions subjects for our comparison process, we crop each reconstructed model on the part representing the valid pixels to take into account in the comparison. After alignment and registration process based on iterative closest point (ICP) with the ground truth model, we compute the absolute depth error. Note that we eliminate examples when the alignment process fails. Finally, we report depth errors evaluated by mean, standard deviation, median, and the average ninety percent most significant error. Note that we report the results obtained on the same data set directly from (Feng et al., 2018a) paper. From Table 2 we can see that our method produces results comparable to the state of the art. The performance of (Feng et al., 2018a) work is slightly better than ours, and we believe that is due to the fact they use part of the BU-3DFE (Yin et al., 2006) data set for training. This data set is acquired using a special sensor, while our model is trained only with generated synthetic data.

We also provide another way of estimating the error between the reconstruction and the ground truth. The criterion is standard deviation $\sigma$ of per-vertex errors between the reconstruction and the ground truth projected on the normals of the ground truth (mean of this error being very close to zero after surface registration). By doing so we evaluate the error between two surfaces in the normal direction instead of distances between points. Since the models do not have the same meshes, we find nearest neighbor for each vertex of the reconstruction. The criteria are defined 


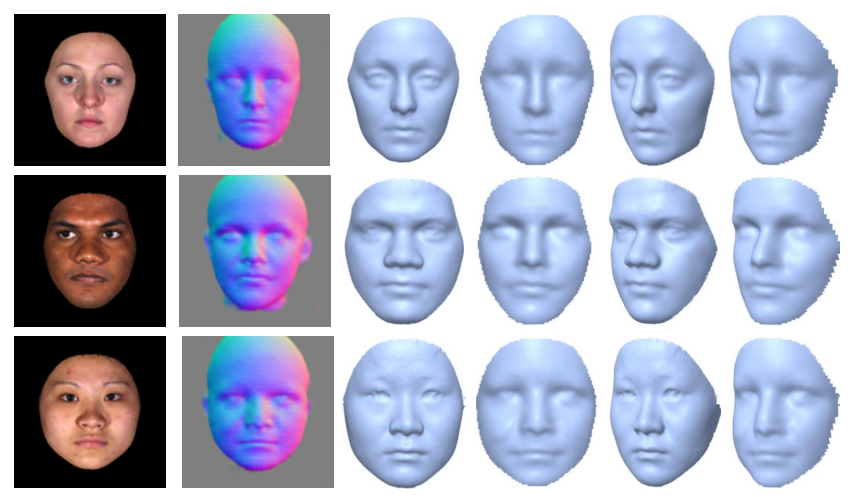

Figure 9: Reconstruction results for three BU-3DFE examples from two different viewpoints. From left to right: input image, normal map $(N)$, front-view ground-truth model, front-view reconstructed model, slide-view ground-truth model and side-view reconstructed model.

\begin{tabular}{|c|c|c|c|c|c|c|}
\hline Method & Mean & Std & Median & $90 \%$ largest & $\bar{e}$ & $\sigma$ \\
\hline (Kemelmacher-Shlizerman and Basri, 2010) & 3.89 & 4.14 & 2.94 & 7.34 & N/A & N/A \\
\hline (Zhu et al., 2015) & 3.85 & 3.23 & 2.72 & 6.82 & N/A & N/A \\
\hline (Richardson et al., 2017) & 3.61 & 2.99 & 2.72 & 6.82 & N/A & N/A \\
\hline (Sela et al., 2017) & 3.51 & 2.69 & 2.65 & 6.59 & N/A & N/A \\
\hline (Feng et al., 2018a $)$ & $\mathbf{2 . 7 8}$ & 2.04 & $\mathbf{1 . 7 3}$ & $\mathbf{5 . 3 0}$ & N/A & N/A \\
\hline Ours & 3.04 & $\mathbf{1 . 7 8}$ & 2.62 & 5.48 & 0.10 & 2.18 \\
\hline
\end{tabular}

Table 2: Quantitative comparison on the BU-3DFE (Yin et al., 2006) data set. Lowers are better

as follows:

$$
\begin{gathered}
\bar{e}=\frac{1}{M} \sum_{k=1}^{M}\left(\mathbf{a}_{k}-\mathbf{b}_{k}\right) \mathbf{n}_{i} \\
V=\frac{1}{M-1} \sum_{k=1}^{M}\left(\left(\mathbf{a}_{k}-\mathbf{b}_{k}\right) \mathbf{n}_{i}-\bar{e}\right)^{2} \\
\sigma=\sqrt{\frac{1}{N} \sum_{k=1}^{E} V_{k}}
\end{gathered}
$$

where $N$ is the total number of examples in the BU3DFE (Yin et al., 2006) data set, $\mathrm{M}$ is the total number of vertices per model. $\mathbf{a}_{k}$ and $\mathbf{b}_{k}$ are the coordinates of vertices from the estimated and ground truth models respectively and $\mathbf{n}_{i}$ is the normals coordinates from the ground truth model. The values of this calculation are reported in the sixth and seventh column of the Table 2

\section{CONCLUSION}

In this work, we have presented a hybrid 3D face reconstruction approach composed of both learning and geometric based methods. The first stage of our main block is an image-to-image translation network that produces normal surface map $(N)$ and gradient magnitude map $(W)$ from a facial input image.
The second stage is integration of normals based on weighted least squares, which uses our network outputs to generate the depth facial map. Our deep learning model has been trained on a fully synthetic facial data set.

We have performed three experiments to evaluate our pipeline performance: first, we show that the neural network generates accurate maps of normals. Next, experiments confirm the effectiveness of using the $\mathrm{W}$ map as a weight during the reconstruction step to resolve discontinuous boundary artifacts. In the final experiment, we demonstrate that the proposed framework achieves the state-of-the-art performance in $3 \mathrm{D}$ face reconstruction. We also propose a new error calculation method, which, we think, is more representative for this type of evaluation. Using this criterion with explicitly written equations avoids any ambiguity on how exactly the evaluation is done and what is the meaning of the obtained value.

Two loss functions $L_{1}$ and $L_{2}$ have been tested as a pixel loss for network training. $L_{2}$ shows a better performance in fine detail reconstruction. And yet some facial features of generated normal maps are still slightly blurred. One possible improvement would be to increase the network complexity and to modify the output layers in order to get sharp details.

Despite the robust performance in many cases, our method has a certain number of limitations. The head 
generator does not include facial expressions and has limited age range. But these limitations can be overcome by using a better synthetic data generator, it is not a fundamental limitation of the proposed method. Furthermore this method is generic and can be applied to any kind of 3D object reconstruction if the right data generator is available. Synthetic data sets allow us to train complex models with virtually unlimited data supply. It accelerates training process by drastically reducing data markup step. On the other hand synthetic data can introduce certain biases in the learning process and therefore one should try to make the generator as photo-realistic as possible. One way of doing that is using GAN architectures in combination of classical 3D rendering.

\section{REFERENCES}

Abate, A. F., Nappi, M., Riccio, D., and Sabatino, G. (2007). 2d and 3d face recognition: A survey. Pattern Recognition Letters, 28(14):1885-1906.

Agarwal, S., Mierle, K., and Others. Ceres solver. http: //ceres-solver.org

Agrawal, A., Raskar, R., and Chellappa, R. (2006). What is the range of surface reconstructions from a gradient field? In European conference on computer vision, pages 578-591. Springer.

Amberg, B., Knothe, R., and Vetter, T. (2008). Expression invariant $3 \mathrm{~d}$ face recognition with a morphable model. In Automatic Face \& Gesture Recognition, 2008. FG'08. 8th IEEE International Conference on, pages 1-6. IEEE.

Bansal, A., Russell, B., and Gupta, A. (2016). Marr revisited: $2 \mathrm{~d}-3 \mathrm{~d}$ alignment via surface normal prediction. In Proceedings of the IEEE conference on computer vision and pattern recognition, pages 5965-5974.

Bas, A., Smith, W. A., Bolkart, T., and Wuhrer, S. (2016). Fitting a 3d morphable model to edges: A comparison between hard and soft correspondences. In Asian Conference on Computer Vision, pages 377-391. Springer.

Blanz, V. and Vetter, T. (1999). A morphable model for the synthesis of $3 \mathrm{~d}$ faces. In Proceedings of the 26th annual conference on Computer graphics and interactive techniques, pages 187-194. ACM Press/AddisonWesley Publishing Co.

Blanz, V. and Vetter, T. (2003). Face recognition based on fitting a $3 \mathrm{~d}$ morphable model. IEEE Transactions on pattern analysis and machine intelligence, 25(9):1063-1074.

Booth, J., Antonakos, E., Ploumpis, S., Trigeorgis, G., Panagakis, Y., Zafeiriou, S., et al. (2017). 3d face morphable models in-the-wild. In Proceedings of the IEEE Conference on ComputerVision and Pattern Recognition.

Dai, H., Pears, N., Smith, W. A. P., and Duncan, C. (2017). A $3 \mathrm{~d}$ morphable model of craniofacial shape and tex- ture variation. In The IEEE International Conference on Computer Vision (ICCV).

Dou, P., Shah, S. K., and Kakadiaris, I. A. (2017). End-toend $3 \mathrm{~d}$ face reconstruction with deep neural networks. In Proc. IEEE Conference on Computer Vision and Pattern Recognition, pages 21-26.

Feng, M., Zulqarnain Gilani, S., Wang, Y., and Mian, A. (2018a). 3d face reconstruction from light field images: A model-free approach. In Proceedings of the European Conference on Computer Vision (ECCV), pages 501-518.

Feng, Y., Wu, F., Shao, X., Wang, Y., and Zhou, X. (2018b). Joint 3d face reconstruction and dense alignment with position map regression network. arXiv preprint arXiv: 1803.07835 .

Frankot, R. T. and Chellappa, R. (1988a). A method for enforcing integrability in shape from shading algorithms. IEEE Trans. Pattern Anal. Mach. Intell., 10(4):439-451.

Frankot, R. T. and Chellappa, R. (1988b). A method for enforcing integrability in shape from shading algorithms. IEEE Transactions on pattern analysis and machine intelligence, 10(4):439-451.

Furukawa, Y. and Ponce, J. (2009). Accurate camera calibration from multi-view stereo and bundle adjustment. International Journal of Computer Vision, 84(3):257268.

Ghosh, A., Fyffe, G., Tunwattanapong, B., Busch, J., Yu, X., and Debevec, P. (2011). Multiview face capture using polarized spherical gradient illumination. In ACM Transactions on Graphics (TOG), volume 30, page 129. ACM.

Gonzalez-Mora, J., De la Torre, F., Guil, N., and Zapata, E. L. (2010). Learning a generic $3 d$ face model from $2 \mathrm{~d}$ image databases using incremental structure-frommotion. Image and Vision Computing, 28(7):11171129.

Han, X., Ugail, H., and Palmer, I. (2009). Gender classification based on $3 \mathrm{~d}$ face geometry features using svm. In CyberWorlds, 2009. CW'09. International Conference on, pages 114-118. IEEE.

Harker, M. and Oleary, P. (2015). Regularized reconstruction of a surface from its measured gradient field. Journal of Mathematical Imaging and Vision, 51(1):46-70.

Hsieh, J.-W., Liao, H.-Y. M., Ko, M.-T., and Fan, K.-C. (1995). Wavelet-based shape from shading. Graphical Models and Image Processing, 57(4):343-362.

Hu, L., Ma, C., Luo, L., and Li, H. (2015). Single-view hair modeling using a hairstyle database. ACM Transactions on Graphics (Proceedings SIGGRAPH 2015), 34(4).

Huber, P., Feng, Z.-H., Christmas, W., Kittler, J., and Ratsch, M. (2015). Fitting 3d morphable face models using local features. In Image Processing (ICIP), 2015 IEEE International Conference on, pages 11951199. IEEE.

Hudon, M., Grogan, M., Pagés, R., and Smolić, A. (2018). Deep normal estimation for automatic shading of 
hand-drawn characters. In European Conference on Computer Vision, pages 246-262. Springer.

Kajiya, J. T. and Kay, T. L. (1989). Rendering fur with three dimensional textures. In ACM Siggraph Computer Graphics, volume 23, pages 271-280. ACM.

Karaçalı, B. and Snyder, W. (2003). Reconstructing discontinuous surfaces from a given gradient field using partial integrability. Computer Vision and Image Understanding, 92(1):78-111.

Kemelmacher-Shlizerman, I. and Basri, R. (2010). 3d face reconstruction from a single image using a single reference face shape. IEEE transactions on pattern analysis and machine intelligence, 33(2):394-405.

Klasing, K., Althoff, D., Wollherr, D., and Buss, M. (2009). Comparison of surface normal estimation methods for range sensing applications. In 2009 IEEE International Conference on Robotics and Automation, pages 3206-3211. IEEE.

Kovesi, P. (2005). Shapelets correlated with surface normals produce surfaces. In Tenth IEEE International Conference on Computer Vision (ICCV'05) Volume 1, volume 2, pages 994-1001. IEEE.

Lin, T.-Y., Maire, M., Belongie, S., Hays, J., Perona, P., Ramanan, D., Dollár, P., and Zitnick, C. L. (2014). Microsoft coco: Common objects in context. In European conference on computer vision, pages 740-755. Springer.

Lun, Z., Gadelha, M., Kalogerakis, E., Maji, S., and Wang, R. (2017). 3d shape reconstruction from sketches via multi-view convolutional networks. In 2017 International Conference on 3D Vision (3DV), pages 67-77. IEEE.

Qiu, J., Cui, Z., Zhang, Y., Zhang, X., Liu, S., Zeng, B., and Pollefeys, M. (2019). Deeplidar: Deep surface normal guided depth prediction for outdoor scene from sparse lidar data and single color image. In Proceedings of the IEEE Conference on Computer Vision and Pattern Recognition, pages 3313-3322.

Quéau, Y. and Durou, J.-D. (2015). Edge-preserving integration of a normal field: Weighted least-squares, tv and $1^{\wedge} 1$ approaches. In International Conference on Scale Space and Variational Methods in Computer Vision, pages 576-588. Springer.

Quéau, Y., Durou, J.-D., and Aujol, J.-F. (2018). Normal integration: a survey. Journal of Mathematical Imaging and Vision, 60(4):576-593.

Richardson, E., Sela, M., Or-El, R., and Kimmel, R. (2017). Learning detailed face reconstruction from a single image. In Proceedings of the IEEE Conference on Computer Vision and Pattern Recognition, pages 1259-1268.

Ronneberger, O., Fischer, P., and Brox, T. (2015). U-net: Convolutional networks for biomedical image segmentation. In International Conference on Medical image computing and computer-assisted intervention, pages 234-241. Springer.

Sela, M., Richardson, E., and Kimmel, R. (2017). Unrestricted facial geometry reconstruction using imageto-image translation. arxiv.
Simchony, T., Chellappa, R., and Shao, M. (1990). Direct analytical methods for solving poisson equations in computer vision problems. IEEE transactions on pattern analysis and machine intelligence, 12(5):435446.

Su, W., Du, D., Yang, X., Zhou, S., and Fu, H. (2018). Interactive sketch-based normal map generation with deep neural networks. Proceedings of the ACM on Computer Graphics and Interactive Techniques, 1(1):22.

Sumner, R. W., Schmid, J., and Pauly, M. (2007). Embedded deformation for shape manipulation. In $A C M$ Transactions on Graphics (TOG), volume 26, page 80. $\mathrm{ACM}$.

Terzopoulos, D. (1988). The computation of visible-surface representations. IEEE Transactions on Pattern Analysis \& Machine Intelligence, (4):417-438.

Thies, J., Zollhofer, M., Stamminger, M., Theobalt, C., and Nießner, M. (2016). Face2face: Real-time face capture and reenactment of rgb videos. In Proceedings of the IEEE Conference on Computer Vision and Pattern Recognition, pages 2387-2395.

Tran, A. T., Hassner, T., Masi, I., and Medioni, G. (2017). Regressing robust and discriminative $3 \mathrm{~d}$ morphable models with a very deep neural network. In Computer Vision and Pattern Recognition (CVPR), 2017 IEEE Conference on, pages 1493-1502. IEEE.

Trigeorgis, G., Snape, P., Zafeiriou, S., and Kokkinos, I. (2017). Normal Estimation For "in-the-wild" Faces Using Fully Convolutional Networks. In Computer Vision and Pattern Recognition (CVPR).

Wang, X., Fouhey, D., and Gupta, A. (2015). Designing deep networks for surface normal estimation. In Proceedings of the IEEE Conference on Computer Vision and Pattern Recognition, pages 539-547.

Wang, Y., Bu, J., Li, N., Song, M., and Tan, P. (2012). Detecting discontinuities for surface reconstruction. In Proceedings of the 21st International Conference on Pattern Recognition (ICPR2012), pages 2108-2111. IEEE.

Xie, W., Wang, M., Wei, M., Jiang, J., and Qin, J. (2019). Surface reconstruction from normals: A robust dgpbased disconti-nuity preservation approach. In Proceedings of the IEEE Conference on Computer Vision and Pat-tern Recognition, pages 5328-5336.

Yin, L., Wei, X., Sun, Y., Wang, J., and Rosato, M. J. (2006). A 3d facial expression database for facial behavior research. In 7th international conference on automatic face and gesture recognition (FGR06), pages 211-216. IEEE.

Zhu, X., Lei, Z., Yan, J., Yi, D., and Li, S. Z. (2015). Highfidelity pose and expression normalization for face recognition in the wild. In Proceedings of the IEEE Conference on Computer Vision and Pattern Recognition, pages 787-796.

Zollhöfer, M., Martinek, M., Greiner, G., Stamminger, M., and Süßmuth, J. (2011). Automatic reconstruction of personalized avatars from $3 \mathrm{~d}$ face scans. Computer Animation and Virtual Worlds, 22(2-3):195-202. 